\title{
Little-Parks Oscillations in Hybrid Ferromagnet-Superconductor Systems
}

\author{
A. V. Samokhvalov, ${ }^{1}$ A. S. Mel'nikov, ${ }^{1}$ J-P. Ader, ${ }^{2}$ and A. I. Buzdin ${ }^{2}$ \\ ${ }^{1}$ Institute for Physics of Microstructures, Russian Academy of Sciences, 603950, Nizhny Novgorod, Russia \\ ${ }^{2}$ Institut Universitaire de France and Université Bordeaux I; CNRS; CPMOH, F-33405 Talence, France
}

(Dated: November 1, 2018)

\begin{abstract}
On the basis of of linearized Usadel equations we consider superconductivity nucleation in multiply connected mesoscopic superconductor/ferromagnet hybrids such as thin-walled superconducting cylinders placed in electrical contact with a ferromagnetic metal. We study the interplay between the oscillations of $T_{c}$ due to the Little-Parks effect and the oscillations due to the exchange field. We demonstrate that the exchange field provokes the switching between the superconducting states with different vorticities and this may result in the increase the critical temperature of the superconducting transition in the magnetic field. Moreover we analyse the influence of the $\mathrm{S} / \mathrm{F}$ transparency on the realisation of the states with higher vorticities.

PACS numbers: 74.25.Dw, 74.45.+c, 74.78.Na
\end{abstract}

\section{INTRODUCTION}

Little-Parks effect, $\frac{1}{1}$ i.e., the oscillations of the critical temperature $T_{c}$ of multiply-connected superconducting samples in an applied magnetic field $H$, is one of the striking phenomena demonstrating coherent nature of the superconducting state. Nevertheless, such oscillatory phase transition line $T_{c}(H)$ was shown to be inherent to simply connected mesoscopic samples with the lateral size of the order of several coherence lengths $\xi^{2}, \underline{3}, \underline{4}$ and to hybrid ferromagnet (F)-superconductor $(\mathrm{S})$ systems with magnetic dots or domains $\frac{5.6,7.8}{}$, that create a "magnetic template" for nucleation of the superconducting order parameter. These $T_{c}(H)$ oscillations reflect the switching between vortex states characterized by different winding numbers and are associated with the orbital effect $\frac{9}{-}$.

Another mechanism of switching between the superconducting states with different vorticities in multiply connected hybrid S/F structures such as thin-walled superconducting shell placed in electrical contact with a ferromagnetic cylinder was suggested recently in 10 . This mechanism is caused by the exchange interaction and associated with the damped-oscillatory behavior of the Cooper pair wave function in a ferromagnet $\underline{\underline{11}}$. It was shown that under certain conditions the exchange interaction can stimulate the superconducting states with a nonzero vorticity in the absence of external magnetic field. The interplay between the exchange and orbital effects may result in a subsequent switching between the states with different vorticities, as the $\mathrm{F}$ core radius increases. An obvious consequence of these transitions between the states with different $L$ should be a nonmonotonic dependence of the critical temperature $T_{c}$ on the $\mathrm{F}$ core radius and exchange field. Similar oscillating behavior of $T_{c}$ on a ferromagnetic layer thickness has been predicted for layered S/F structures $\underline{12,13}$. Note, that the unusual ground states with spontaneously formed vortices in multiply-connected $\mathrm{S} / \mathrm{F}$ hybrids resemble the behavior of Josephson $\pi$-junction $\frac{14,15,16}{16}$ with a step-like change in the F layer thickness $17,18,19$.

It is the purpose of this paper to study the influence of an external magnetic field on the proximity induced switching between the vortex states. We focus on the behavior of critical temperatures for superconducting states with different vorticities and, thus, we study the LittleParks effect affected by the exchange interaction.

The paper is organized as follows. In Sec. Iw we briefly discuss the basic equations. In Sec. III we study the switching between different vortex states for two model $\mathrm{S} / \mathrm{F}$ systems placed in the external magnetic field. The first system consists of a thin-walled superconducting cylindrical shell surrounding a cylinder of a ferromagnetic metal. As a second example we consider a cylindrical cavity in a bulk ferromagnet covered by a thin layer of superconducting material. For both cases we assume that there is a good electrical contact between the $\mathrm{F}$ and $\mathrm{S}$ regions, to assure a rather strong proximity effect. We summarize our results in Sec. IV]

\section{MODEL}

The calculations of the second-order superconducting phase transition temperature $T_{c}$ are based on the linearized Usadel equations 20 for the averaged anomalous Green's functions $F_{f}$ and $F_{s}$ for the $\mathrm{F}$ and $\mathrm{S}$ regions, respectively (see Ref $\stackrel{11}{=}$ for details). The superconducting critical temperature $T_{c}$ and exchange field $h$ is assumed to satisfy the dirty-limit conditions $T_{c} \tau \ll 1$ and $h \tau \ll 1$, where $\tau$ is the elastic electron-scattering time.

In the $\mathrm{F}(\mathrm{S})$ region the linearized Usadel equations take the form

$$
\begin{aligned}
& -\frac{D_{f}}{2}\left(\nabla+\frac{2 \pi i}{\Phi_{0}} \mathbf{A}\right)^{2} F_{f} \\
& \quad+(|\omega|+\imath h \operatorname{sgn} \omega) F_{f}=0 \\
& -\frac{D_{s}}{2}\left(\nabla+\frac{2 \pi i}{\Phi_{0}} \mathbf{A}\right)^{2} F_{s}+|\omega| F_{s}=\Delta(\mathbf{r}) .
\end{aligned}
$$

Here $D_{f}$ and $D_{s}$ are the diffusion constants in the ferromagnet and superconductor, respectively, $\omega=(2 n+$ 
1) $\pi T_{c}$ is a Matsubara frequency at the temperature $T_{c}$, and $\Phi_{0}=\pi \hbar c /|e|$ is the flux quantum. Hereafter we consider only a step-like exchange field profile and, thus, neglect both a reduction of the magnetization in the ferromagnet and magnetization leakage into the superconductor. Such assumption is justified above $T_{c}$ due to the local nature of the exchange interaction. Calculating the $T_{c}$ value itself we can assume the superconducting order parameter to be vanishingly small and, thus, it is natural to neglect the spreading of the magnetic moment into the superconductor (see, e.g., 21,22 ).

The superconducting critical temperature $T_{c}$ is determined from the self-consistency condition for the gap function:

$$
\Delta(\mathbf{r}) \ln \frac{T_{c}}{T_{c 0}}+\pi T_{c} \sum_{\omega}\left(\frac{\Delta(\mathbf{r})}{|\omega|}-F_{s}(\mathbf{r}, \omega)\right)=0 .
$$

Equations (11), (2) must be supplemented with the boundary condition at the outer surfaces

$$
\partial_{\mathbf{n}} F_{f, s}=0
$$

and at the interface between the F and S metals: ${ }^{23}$

$$
\sigma_{s} \partial_{\mathbf{n}} F_{s}=\sigma_{f} \partial_{\mathbf{n}} F_{f} ; \quad F_{s}=F_{f}-\gamma_{b} \xi_{n} \partial_{\mathbf{n}} F_{f}
$$

Here $\xi_{s(n)}=\sqrt{D_{s(f)} / 2 \pi T_{c 0}}$ is the superconducting (normal-metal) coherence length, $\sigma_{f}$ and $\sigma_{s}$ are the normal-state conductivities of the $\mathrm{F}$ and $\mathrm{S}$ metals, $\gamma_{b}$ is related to the $\mathrm{S} / \mathrm{F}$ boundary resistance $R_{b}$ per unit area through $\gamma_{b} \xi_{s}=R_{b} \sigma_{f}$, and $\partial_{\mathbf{n}}$ denotes a derivative taken in the direction to the outer normal to the $\mathrm{S}$ surfaces, i.e., the vector $\mathbf{n}$ is directed from the $\mathrm{S}$ to the $\mathrm{F}$ metal at the $\mathrm{S} / \mathrm{F}$ interface. For the sake of simplicity we assume $h \gg \pi T_{c 0}$. According to the equations (11),(2), (3) there is a symmetry $F_{f, s}(\omega)=F_{f, s}^{*}(-\omega)$, so that we can treat only positive $\omega$ values.

We consider here a generic example of hybrid S/F systems with a cylindrical symmetry: a thin-walled superconducting hollow cylinder placed in an electrical contact with a ferromagnet (see Fig. 1). The ferromagnetic material is assumed to fill either the internal (Fig. 1a) or external (Fig. 10) region of the system. We choose cylindrical coordinates $(r, \theta, z)$ as it is shown in Fig. 1 take the gauge $\mathbf{A}=\left(0, A_{\theta}, 0\right)$, and look for a homogeneous along $z$ solution of the equations (11), (2), (3) characterized by certain angular momentum $L$ :

$$
\Delta(\mathbf{r})=\Delta(r) \mathrm{e}^{\imath L \theta}, \quad F_{f, s}(\mathbf{r})=f_{f, s}(r) \mathrm{e}^{\imath L \theta} .
$$

The vorticity parameter $L$ just coincides with the angular momentum of the Cooper pair wave function.

Certainly, the magnetization $\mathbf{M}$ inside the $F$ region makes contribution to the vector potential $\mathbf{A}$ :

$$
\mathbf{B}=\operatorname{rot} \mathbf{A}, \quad \mathbf{B}=\mathbf{H}+4 \pi \mathbf{M},
$$

and can modify the conditions of superconductivity nucleation in the $\mathrm{S}$ shell. Choosing the magnetization direction along the $z$-axis $\mathbf{M}=M \mathbf{z}_{0}$, we can roughly estimate the relevant change in the total magnetic flux as a)

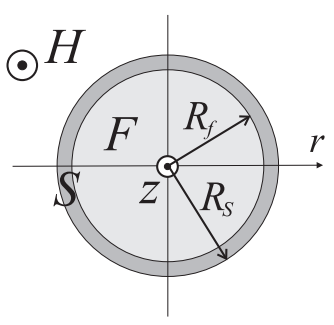

b)

FIG. 1: The cross section of the hybrid S/F systems under consideration: a) thin-walled superconducting shell (S) around a ferromagnetic cylinder $(\mathrm{F})$; b) cylindrical cavity in a bulk ferromagnet $(\mathrm{F})$ covered by a thin layer of superconducting material (S). Here $R_{f}$ is the radius of the $\mathrm{F}$ core (cavity), and $R_{s}$ is the outer (inner) radius of the $\mathrm{S}$ shell; $(r, \theta, z)$ is the cylindrical coordinate system. External magnetic field $\mathbf{H}$ applied along the $z$ axis.

follows: $\Phi_{M} \sim 4 \pi^{2} R_{f}^{2} M$. In principle, this change in the magnetic flux $\Phi_{M}$ modifies the period of the standard Little-Parks oscillations and breaks the symmetry of the $T_{c}(H)$ dependence with respect to the external magnetic field inversion: $T_{c}(H) \neq T_{c}(-H)$. However, for typical parameters $M \sim 10^{2} \mathrm{G}, T \sim 10 \mathrm{~K}, D_{s} \sim 10 \mathrm{~cm}^{2} / \mathrm{s}$ and $R_{f}$ of order of several $\xi_{f} \sim 10 \mathrm{~nm}$ lengths we get $\Phi_{M} \ll \Phi_{0}$, and the asymmetry of the Little-Parks curve $T_{c}(H)$ may be neglected. The above effect of the magnetization can be also weakened provided we decrease the size of the $\mathrm{S} / \mathrm{F}$ system along the $z$-axis, going over to the case of a thin $\mathrm{S} / \mathrm{F}$ disk when the field $B$ is suppressed due to the demagnetization factor. Note that choosing the magnetization direction in the plane perpendicular to the cylinder axis we can get rid of the above magnetic flux correction completely because of the absence of the magnetization induced field component along the cylinder axis. These simple estimates allow us to exclude the effect of magnetization on $T_{c}$ assuming that $B \simeq H$ and $A_{\theta}=r H / 2$.

The Usadel equations (11),(2) take the form:

$$
\begin{gathered}
-\frac{D_{f}}{2}\left[\frac{1}{r} \partial_{r}\left(r \partial_{r} f_{f}\right)-\left(\frac{L}{r}+\frac{r}{2 a_{H}}\right)^{2} f_{f}\right] \\
+\imath h f_{f}=0, \\
-\frac{D_{s}}{2}\left[\frac{1}{r} \partial_{r}\left(r \partial_{r} f_{s}\right)-\left(\frac{L}{r}+\frac{r}{2 a_{H}}\right)^{2} f_{s}\right] \\
+\omega f_{s}=\Delta,
\end{gathered}
$$

where $a_{H}=\sqrt{\Phi_{0} / 2 \pi H}$ is the magnetic length. An appropriate self-consistency equation (3) can be rewritten as follows:

$$
\Delta \ln \frac{T_{c}}{T_{c 0}}+2 \pi T_{c} \sum_{\omega>0}\left(\frac{\Delta}{\omega}-\operatorname{Re} f_{s}(\omega)\right)=0 .
$$




\section{VORTEX STATES IN THIN-WALLED SUPERCONDUCTING CYLINDER}

Now we proceed with calculations of the critical temperature dependence on the external magnetic field for two examples of multiply connected mesoscopic hybrid $\mathrm{S} / \mathrm{F}$ systems. The first one is a ferromagnetic cylindrical filament (core) surrounded by a thin-walled superconducting shell (see Fig. 10). The second one is a cylindrical cavity in a bulk ferromagnet covered by a thin layer of superconducting material (see Fig. 1b). External magnetic field $\mathbf{H}$ is assumed to be parallel to the cylinder axis $\left(\mathbf{H}=H \mathbf{z}_{0}\right)$ for the both cases.

\section{A. Ferromagnetic filament covered by a cylindrical superconducting shell}

Consider a superconducting cylindrical shell of a thickness $W=R_{s}-R_{f} \ll R_{f}$ surrounding a cylinder (core) of a ferromagnetic metal. Here $R_{f}$ is the radius of the $\mathrm{F}$ core, and $R_{s}$ is the outer radius of the $\mathrm{S}$ shell (see Fig. (1)). Naturally, to observe a pronounced influence of the proximity effect on the transition temperature, the thickness of the S shell $W$ must be smaller than the superconducting coherence length $\xi_{s}$.

The solution of Eqn. (8) in the F cylinder can be expressed via the confluent hypergeometric function of the first kind (Kummer's function) $F(a, b, z)^{24}$

$$
f_{f}(r)=C \mathrm{e}^{-\phi / 2} \phi^{|L| / 2} F\left(a_{L}, b_{L}, \phi\right),
$$

where $\phi$ is the flux of the external magnetic field $\mathbf{H}$ threading the circle of radius $r$ in the units of the flux quantum $\Phi_{0}$

$$
\phi=2 \pi r A_{\theta} / \Phi_{0}=r^{2} / 2 a_{H}^{2},
$$

and

$$
a_{L}=\frac{|L|+L+1}{2}+i\left(\frac{a_{H}}{\xi_{f}}\right)^{2} ; \quad b_{L}=|L|+1 .
$$

Here $\xi_{f}=\sqrt{D_{f} / h}$ is the characteristic length scale of the order parameter variation in the $\mathrm{F}$ metal. In the dirty limit, the parameter $\xi_{f}$ determines both the length scale of oscillations and the decay length for the Cooper pair wave function in a ferromagnet. The boundary conditions (4) and (5) for Eq. (9) take the form

$$
\left.\frac{d f_{s}}{d r}\right|_{R_{f}}=Q_{L}\left(\phi_{f}\right) f_{s}\left(R_{f}\right),\left.\quad \frac{d f_{s}}{d r}\right|_{R_{s}}=0,
$$

where $\phi_{f}=\pi R_{f}^{2} H / \Phi_{0}$ is the flux of the external magnetic field enclosed in the $\mathrm{F}$ cylinder in the units of flux quantum $\Phi_{0}$, and

$$
\begin{aligned}
Q_{L}\left(\phi_{f}\right) & =\frac{\sigma_{f} / \sigma_{s}}{\gamma_{b} \xi_{n}+R_{f} / \kappa_{L}\left(\phi_{f}\right)}, \\
\kappa_{L}\left(\phi_{f}\right) & =|L|-\phi_{f} \\
& +2 \phi_{f} \frac{a_{L} F\left(a_{L}+1, b_{L}+1, \phi_{f}\right)}{b_{L} F\left(a_{L}, b_{L}, \phi_{f}\right)} .
\end{aligned}
$$
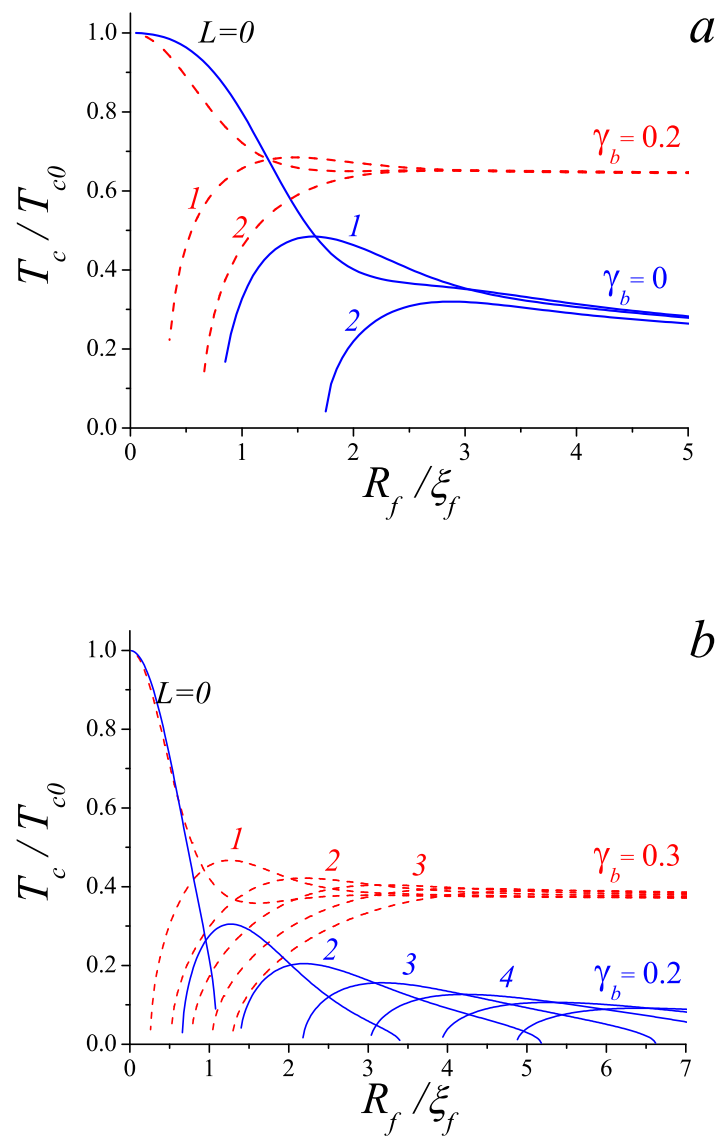

FIG. 2: (Color online) The typical dependences of the critical temperature $T_{c}$ on the $\mathrm{F}$ core radius $R_{f}$ in the absence of the external magnetic field for different values of the interface resistance $\gamma_{b}$. The numbers near the curves denote the corresponding values of vorticity $L$. We choose the parameters (a) $W=0.5 \xi_{s}, \xi_{s} / \xi_{f}=0.1, \xi_{n} / \xi_{f}=4.0, \sigma_{s} / \sigma_{f}=1 ;$ (b) $W=0.5 \xi_{s}, \xi_{s} / \xi_{f}=0.02, \xi_{n} / \xi_{f}=4.0, \sigma_{s} / \sigma_{f}=0.1$.

For $W \ll \xi_{s}$, the variations of the functions $f_{s}(r)$ and $\Delta(r)$ in the superconducting shell are small: $f_{s}(r) \simeq f$, $\Delta(r) \simeq \Delta$. Therefore, we can average Eq. (8) over the thickness of the $\mathrm{S}$ shell, using the boundary conditions (13) to integrate the term $\partial_{r}\left(r \partial_{r} f_{s}\right)$. Finally, we obtain the following expression:

$$
f=\frac{\Delta}{\omega+\frac{D_{s}}{2}\left[\left(\frac{L+\phi_{f}}{R_{f}}\right)^{2}+\frac{Q_{L}\left(\phi_{f}\right)}{W}\right]} .
$$

Substituting the solution (15) into Eq.(9) one obtains a self-consistency equation for the critical temperature $T_{L}$ of the state with a vorticity $L$ :

$$
\ln \frac{T_{L}}{T_{c 0}}=\Psi\left(\frac{1}{2}\right)-\operatorname{Re} \Psi\left(\frac{1}{2}+\Omega_{L}\left(\phi_{f}\right)\right)
$$

where $\Psi$ is the digamma function. The depairing param- 


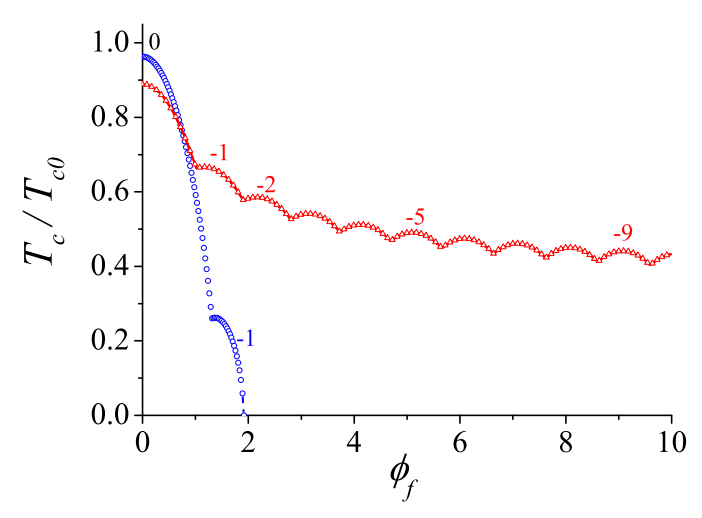

$a$
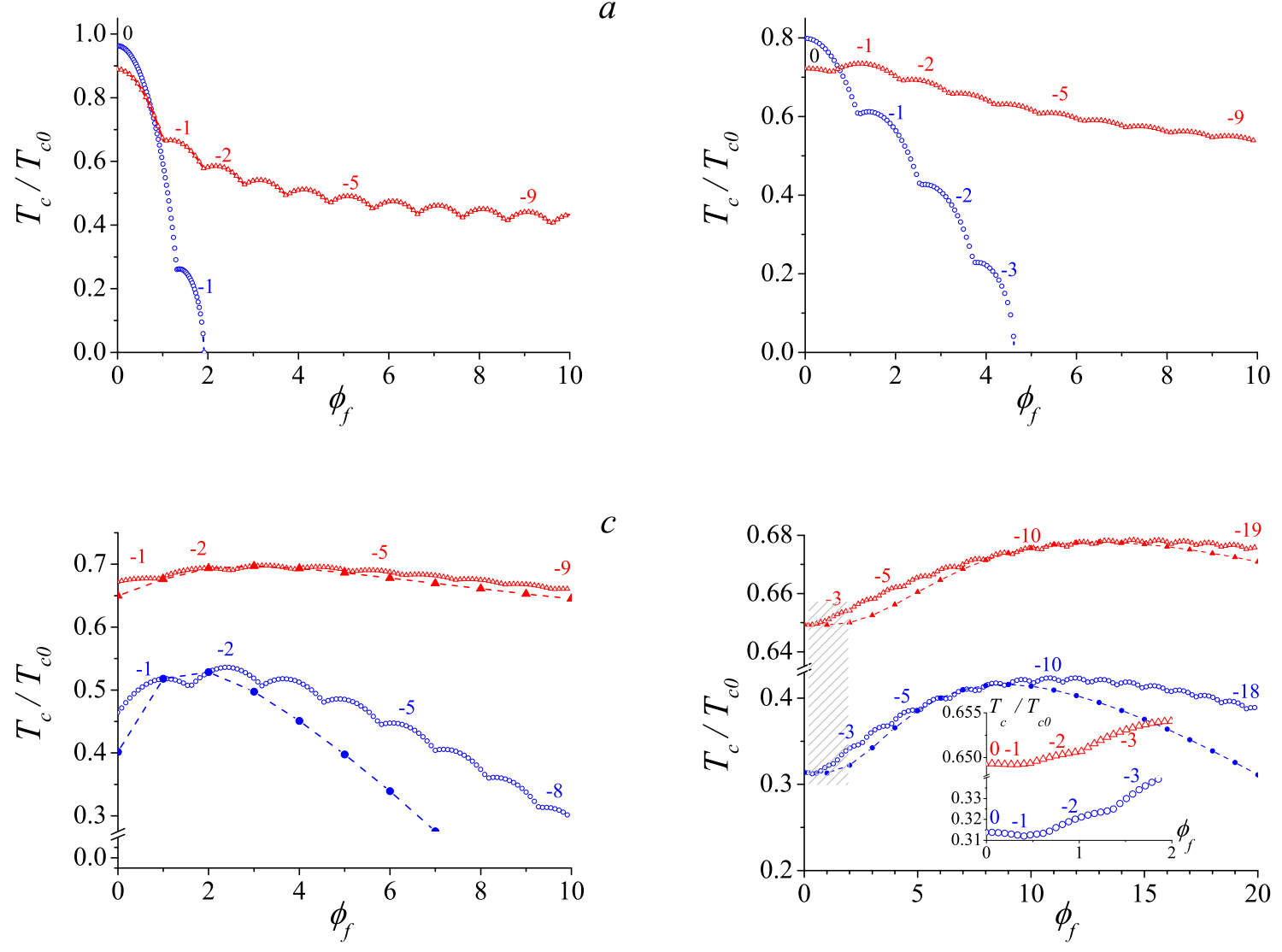

$c$

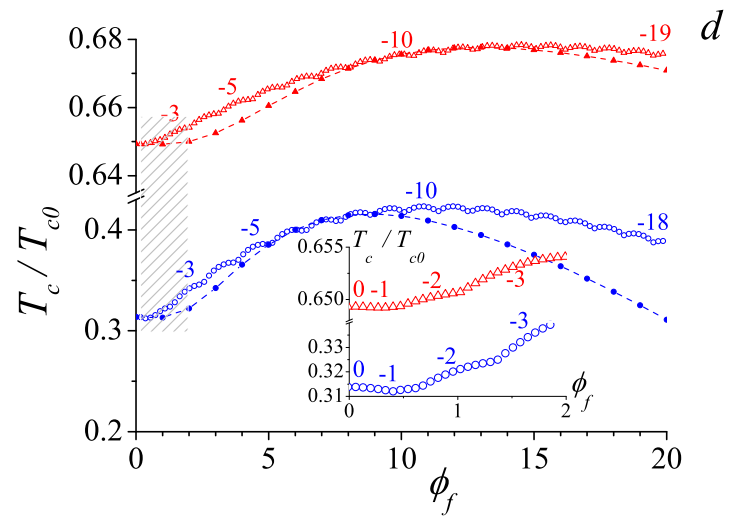

FIG. 3: (Color online) The typical dependences of the critical temperature $T_{c}$ on the external magnetic field $H$ for different values of the interface resistance $\gamma_{b}: \gamma_{b}=0(\circ) ; \gamma_{b}=0.2(\triangle)$. The magnetic field $H$ is measured in the units of the magnetic flux $\phi_{f}$ enclosed in $\mathrm{F}$ cylinder. The numbers near the curves denote the corresponding values of vorticity $L$. Here we choose $W=0.5 \xi_{s} ; \xi_{s} / \xi_{f}=0.1 ; \xi_{n} / \xi_{f}=4.0 ; \sigma_{s} / \sigma_{f}=1$, and different values of the $\mathrm{F}$ cylinder radius $R_{f} / \xi_{f}=(\mathrm{a}) 0.5$, (b) 1 , (c) 2 , (d) 4. The inset in panel (d) gives the zoomed part of the $T_{c}(H)$ line, marked by the shaded box. The dashed lines in panels (c, d) are guides for eye which connect the points corresponding to the $T_{c}$ values found for $\phi_{f}=-L$, when the orbital effect in the depairing parameter (17) is cancelled.

eter of the mode $L$

$$
\Omega_{L}\left(\phi_{f}\right)=\frac{1}{2} \frac{T_{c 0}}{T_{L}} \xi_{s}^{2}\left[\left(\frac{L+\phi_{f}}{R_{f}}\right)^{2}+\frac{Q_{L}\left(\phi_{f}\right)}{W}\right]
$$

is responsible for the superconductivity destruction in the shell in the applied magnetic field $\mathbf{H}$, due to both the orbital and exchange effects. As usual, the critical temperature $T_{c}$ of a superconductivity nucleation in the shell is determined by the maximal value $T_{L}$ :

$$
T_{c}=\max _{L}\left\{T_{L}\right\}
$$

We start our numerical analysis from the case of zero external magnetic field focusing on the effect of the $\mathrm{S} / \mathrm{F}$ interface resistance $R_{b}$ on the behavior of $T_{c}\left(R_{f}\right)$. Taking into account the asymptotic expressions for the Kummer's function $F(a, b, z / a)$ for $|a| \rightarrow \infty$ we obtain the parameter $\kappa_{L}$ in a simplified form:

$$
\kappa_{L}(0)=|L|+u_{f} \frac{I_{|L|+1}\left(u_{f}\right)}{I_{|L|}\left(u_{f}\right)}, \quad u_{f}=\frac{R_{f}}{\xi_{f}}(1+i) .
$$

The states with angular momenta $\pm L$ are degenerated for $H=0$ and have the same critical temperature $T_{c}$.

In Fig. 2 we present examples of dependencies of the critical temperature $T_{c}$ on the $\mathrm{F}$ cylinder radius $R_{f}$ for different values of the $\mathrm{S} / \mathrm{F}$ interface transparency. We see that for a small $\mathrm{F}$ cylinder radius $R_{f} \ll \xi_{f}$ only the state with $L=0$ appears to be energetically favorable. The influence of the proximity effect is weak and the critical temperature $T_{c}$ is close to $T_{c 0}$. For a vortex state with $L \geq 1$ the $T_{c}$ value is suppressed because of a large orbital effect. The increase in the radius $R_{f}$ results in a decrease in $T_{c}$ for the state with $L=0$ and reduce the kinetic energy of supercurrents for $L \geq 1$. At the same time, the damped-oscillatory behavior of the supercon- 
ducting order parameter in a ferromagnet becomes important, since the diameter of the $\mathrm{F}$ cylinder becomes comparable with the period of the order parameter oscillations $\left(\sim \xi_{f}\right)$. As a result, for $R_{f}>\xi_{f}$ the vortex state with $L=1$ becomes more energetically favorable due to the exchange interaction. It is interesting to note that at small $\mathrm{F}$ cylinder radius $\left(R_{f}<\xi_{f}\right)$ the critical temperature of the vortex free mode with $L=0$ decreases with an increase of the interface barrier $\gamma_{b}$, if the condition $\gamma_{b} \xi_{n} R_{f}<\xi_{f}^{2}$ is fulfilled. Such counterintiutive behavior is explained by the enchancement of the pair-breaking role of the exchange field due to the increase in the time of the Cooper pair stay in the $\mathrm{F}$ metal in the presence of the interface barrier ${ }^{11}$.

Figure 3 shows examples of dependences of the critical temperature $T_{c}$ on the external magnetic field $H$, obtained from Eqs. (16), (17), (18) for different values of $\mathrm{F}$ cylinder radius $R_{f}$. Due to the symmetry of the phase boundary $T_{c}(-H)=T_{c}(H)$, we present here the curves for positive values of the external field $H$ only. The phase boundary exhibits Little-Parks oscillations, indicating transitions between the states with different angular momenta $L \rightarrow L \pm 1$ of the superconducting order parameter. For a small F cylinder radius (Fig. 3a,b) the influence of the exchange interaction is weak and the $T_{c}(H)$ phase boundary exhibits undamped quasiperiodic oscillations as function of magnetic field and resembles the $T_{c}(H)$ curve for a mesoscopic disk-shaped superconductor ${ }^{2,4}$. It means that the $\mathrm{S}$ ring induces superconductivity in the thin $\mathrm{F}$ filament due to proximity, and the behavior of the S/F hybrid system under consideration is similar to the one for a superconducting cylinder (or disk) with an inhomogeneous order parameter.

With an increase in the $\mathrm{F}$ cylinder radius $R_{f}$ one can clearly observe a shift of the main $T_{c}$ maximum towards nonzero $H$ values. To explain this shift we note that the highest critical temperature for a given magnetic flux $\phi_{f}$ corresponds to the states with the angular momentum $L$ close to the integer part of the $-\phi_{f}$ value. Exactly at the points $\phi_{f}=-L$ the orbital term in the depairing parameter (17) is cancelled and the dependendence of $T_{c}$ vs $\phi_{f}$ at these points is determined only by the exchange effect. On the other hand, for $R_{f} \gtrsim \xi_{f}$ this exchange part of the depairing parameter is minimal and the corresponding $T_{c}$ is maximal for a state with a nonzero vorticity (see Fig. 2). Thus, the main $T_{c}$ maximum shifts to a certain nonzero $\phi_{f}$ value. To support this explanation we have performed simulations of the critical temperature $T_{c}$ for the points $\phi_{f}=-L$, i.e. when the orbital effect is cancelled. The results are shown in panels (c, d) of Fig. 3 by dashed lines. These curves describe a large scale (as compared to $\left.\phi_{f}\right)$ behavior of the phase boundary $T_{c}(H)$ and clearly demonstrate the shift of the main $T_{c}$ maximum. With the increase in the $\mathrm{F}$ cylinder radius $R_{f}$ and the interface barrier $\gamma_{b}$ this main $T_{c}$ maximum shift appears to increase.

Note, that a similar shift of the main $T_{c}$ maximum has been observed in mesoscopic $\mathrm{S} / \mathrm{F}$ hybrid structures without the proximity effect $\frac{5,8}{}$ and in the quasi-twodimensional organic conductors 25,26 . In the latter case, an applied magnetic field compensates the exchange fields of the paramagnetic ions and neutralizes the destructive action of these fields 27 .

\section{B. Cylindrical cavity in a bulk ferromagnet covered by a superconductor}

As a second example we consider a superconducting shell of a thickness $W=R_{f}-R_{s}$ covering a cylindrical cavity of the radius $R_{f}$ in a ferromagnetic material (see Fig. 1]). For simplicity, we consider only rather thin S shells with $W<\xi_{s}$ which allows us to assume the variations of the functions $f_{s}(r)$ and $\Delta(r)$ in the superconductor to be small. The solution of Eqn. (8) in F metal can be expressed via the confluent hypergeometric function of the second kind $U(a, b, z)^{24}$,

$$
f_{f}(r)=C \mathrm{e}^{-\phi / 2} \phi^{|L| / 2} U\left(a_{L}, b_{L}, \phi\right),
$$

where the flux $\phi$, parameters $a_{L}$ and $b_{L}$ are determined by the expressions (11) and (12). The boundary conditions (4) and (5) for Eq. (9) take the form

$$
\left.\frac{d f_{s}}{d r}\right|_{R_{s}}=0,\left.\quad \frac{d f_{s}}{d r}\right|_{R_{f}}=-\tilde{Q}_{L}\left(\phi_{f}\right) f_{s}\left(R_{f}\right),
$$

where $\phi_{f}=\pi R_{f}^{2} H / \Phi_{0}$ is the flux of the external magnetic field enclosed in the cavity in the units of the flux quantum $\Phi_{0}$, and

$$
\begin{aligned}
\tilde{Q}_{L}\left(\phi_{f}\right) & =\frac{\sigma_{f} / \sigma_{s}}{\gamma_{b} \xi_{n}-R_{f} / \tilde{\kappa}_{L}\left(\phi_{f}\right)}, \\
\tilde{\kappa}_{L}\left(\phi_{f}\right) & =|L|-\phi_{f} \\
& -2 \phi_{f} \frac{a_{L} U\left(a_{L}+1, b_{L}+1, \phi_{f}\right)}{U\left(a_{L}, b_{L}, \phi_{f}\right)} .
\end{aligned}
$$

Assuming that the variations of the functions $f_{s}(r)$ and $\Delta(r)$ in the superconducting shell are small $\left(f_{s}(r) \simeq f\right.$, $\Delta(r) \simeq \Delta$ ) we can average Eq. (9) over the thickness of the $\mathrm{S}$ shell, using the boundary conditions (21) to integrate the term $\partial_{r}\left(r \partial_{r} f_{s}\right)$. Substitution of the solution

$$
f=\frac{\Delta}{\omega+\frac{D_{s}}{2}\left[\left(\frac{L+\phi_{f}}{R_{f}}\right)^{2}+\frac{\tilde{Q}_{L}\left(\phi_{f}\right)}{W}\right]}
$$

into the self-consistency equation (9) results in the equation for the critical temperature $T_{L}$ of the state with a vorticity $L$ (15), where the depairing parameter $\Omega_{L}$ of the mode $L$ is determined by the following expression:

$$
\Omega_{L}\left(\phi_{f}\right)=\frac{1}{2} \frac{T_{c 0}}{T_{L}} \xi_{s}^{2}\left[\left(\frac{L+\phi_{f}}{R_{f}}\right)^{2}+\frac{\tilde{Q}_{L}\left(\phi_{f}\right)}{W}\right] .
$$

Figure 4 shows examples of dependencies of the critical temperature $T_{c}$ on the external magnetic field $H$, 

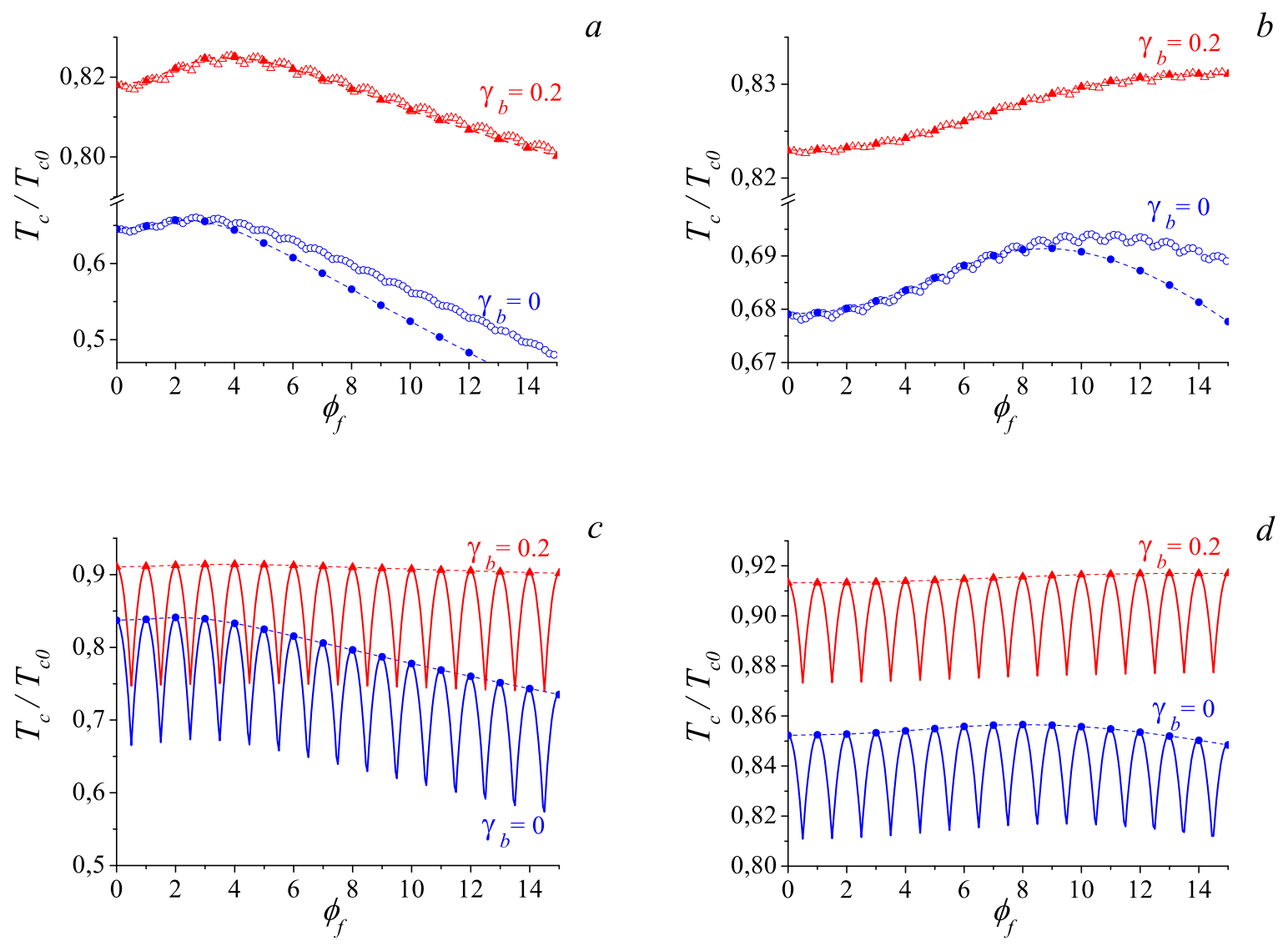

FIG. 4: (Color online) The typical dependencies of the critical temperature $T_{c}$ on the external magnetic field $H$ for two values of the cylindrical cavity radius $R_{f}\left(R_{f} / \xi_{f}=2\right.$ (panels (a, c); $R_{f} / \xi_{f}=4$ (panels b, d) ) and different values of the the interface resistance $\gamma_{b}$. The magnetic field $H$ is measured in the units of the magnetic flux $\phi_{f}$ enclosed in the cavity. Here we choose $W=0.5 \xi_{s} ; \xi_{n} / \xi_{f}=4.0$. The panels $(\mathrm{a}, \mathrm{b})$ show the case of the S/F system with a rather small ratio $\xi_{s} / \xi_{f}=0.1$ and $\sigma_{s} / \sigma_{f}=2$. The panels $(\mathrm{c}, \mathrm{d})$ show the case of the $\mathrm{S} / \mathrm{F}$ system with $\xi_{s} / \xi_{f}=1$ and $\sigma_{s} / \sigma_{f}=40$. The dashed lines are guides for eye which connect the points corresponding to the $T_{c}$ values found for $\phi_{f}=-L$, when the orbital effect in the depairing parameter (24) is cancelled.

obtained from Eqs. (16), (22), (24) for two values of the cylindrical cavity radius $R_{f}$. Similarly to the previous subsection this phase boundary resembles the $T_{c}(H)$ curve for the Little-Parks oscillations. Slow envelopes of the oscillating curve clearly demonstrate the shift of the main $T_{c}$ maximum towards nonzero $H$ values.

\section{SUMMARY}

To sum up, we have analysed the behavior of the LittleParks oscillations of the critical temperature $T_{c}$ on an external magnetic field $H$ in multiply connected $\mathrm{S} / \mathrm{F}$ systems affected by the exchange interaction. As an example, we have considered mesoscopic thin-walled superconducting cylindrical shell placed in electrical contact with a ferromagnet. The phase-transition line $T_{c}(H)$ and order parameter structure have been studied on the basis of linearized Usadel equations. We have demonstrated that the exchange field provokes the switching between the superconducting states with different vorticities: the interplay between the oscillations of $T_{c}$ due to the LittleParks effect and the oscillations due to the exchange field results in breaking of the strict periodicity of the $T_{c}(H)$ dependence. We have also observed a slow modulation of the amplitude of the quasiperiodic $T_{c}(H)$ oscillations. With an increase in the superconducting shell radius the envelope of the oscillating phase transition line $T_{c}(H)$ exhibits a shift of the main $T_{c}$ maximum to finite external magnetic field values. This shift is explained by the increase in the critical temperature of the superconducting states with nonzero vorticities due to the exchange interaction. The above effects strongly depend on the S/F interface barrier strength. We have shown that a decrease in the $\mathrm{S} / \mathrm{F}$ transparency may stimulate the states with higher vorticities. 


\section{ACKNOWLEDGMENTS}

This work was supported, in part, by the Russian Foundation for Basic Research, by International Ex- change Program of Universite Bordeaux I, by French ANR project "ELEC-EPR", by the "Dynasty" Foundation, and by the program of LEA Physique Theorique et Matiere Condensee.
1 W. A. Little and R. D. Parks, Phys. Rev. Lett. 9, 9 (1962); R. D. Parks and W. A. Little, Phys. Rev. 133, A97 (1964).

2 O. Buisson, P. Gandit, R. Rammal, Y. Y. Wang, and B. Pannetier, Phys. Lett. 150, 36 (1990).

3 V. Bruyndoncx, J. G. Rodrigo, T. Puig, L. Van Look, V. V. Moshchalkov, and R. Jonckheere, Phys. Rev. B 60, 4285 (1999).

4 H. T. Jadallah, J. Rubinstein, and P. Sternberg, Phys. Rev. Lett. 82, 2935 (1999).

${ }^{5}$ M. Lange, M. J. Van Bael, Y. Bruynseraede, and V. V. Moshchalkov, Phys. Rev. Lett. 90, 197006 (2003).

6 A. Yu. Aladyshkin, A. S. Mel'nikov, D. A. Ryzhov, J. Phys.: Condens. Matt. 15, 6591 (2003).

7 A. Yu. Aladyshkin, A. I. Buzdin, A. A. Fraerman, A. S. Mel'nikov, D. A. Ryzhov, and A. V. Sokolov, Phys. Rev. B 68, 184508 (2003).

8 N. Schildermans, A. Yu. Aladyshkin, A. V. Silhanek, J. Van de Vondel, and V. V. Moshchalkov, Phys. Rev. B 77, 214519 (2008).

9 V. Ginzburg, Zh .Eksp. Teor. Phys. 31, 202 (1956) [Sov. Phys. JETP 4, 153 (1956)].

10 A. V. Samokhvalov, A. S. Melnikov, and A. I. Buzdin, Phys. Rev. B 76, 184519 (2007).

11 A. I. Buzdin, Rev. Mod. Phys. 77, 935 (2005).

12 A. I. Buzdin and M. V. Kuprianov, Pisma Zh. Eksp. Teor. Fiz. 52, 1089 (1990). [JETP Lett. 52, 487 (1990)].

13 Z. Radovic, M. Ledvij, L. Dobrosavljevic-Grujic, A. I. Buzdin, and J. R. Clem, Phys. Rev. B 44, 759 (1991).

14 A. I. Buzdin, L. N. Bulaevskii, and S. V. Panyukov, Pisma Zh. Eksp. Teor. Fiz. 35, 147 (1982) [JETP Lett. 35, 178 (1982)].
15 A. I. Buzdin and M. V. Kuprianov, Pisma Zh. Eksp. Teor. Fiz. 5, 308 (1991) [JETP Lett. 53, 321 (1991)].

16 V. V. Ryazanov, V. A. Oboznov, A. Yu. Rusanov, A. V. Veretennikov, A. A. Golubov, and J. Aarts, Phys. Rev. Lett. 86, 2427 (2001).

17 L. N. Bulaevsky, V. V. Kuzii, and A. A. Sobyanin, Solid State Commun. 25, 1053 (1978).

18 M. Weides, M. Kemmler, E. Goldobin, H. Kohlstedt, R. Waser, D. Koelle, and R. Kleiner, Phys. Rev. Lett. 97, 247001 (2006).

19 S. M. Frolov, D. J. Van Harlingen, V. V. Bolginov, V. A. Oboznov, and V. V. Ryazanov, Phys. Rev. B 74, 020503(R) (2006).

${ }^{20}$ L. Usadel, Phys. Rev. Lett. 95, 507 (1970).

21 V. N. Krivoruchko and E. A. Koshina, Phys. Rev. B 66, 014521 (2002).

22 F. S. Bergeret, A. F. Volkov, and K. B. Efetov, Phys. Rev. B 69, 174504 (2004).

23 M. Yu. Kuprianov and V. F. Lukichev, Zh. Eksp. Teor. Fiz. 94, 139 (1988) [Sov. Phys. JETP 67, 1163 (1988)].

24 Handbook of Mathematical Functions, Natl. Bur. Stand. Appl. Math. Ser. No. 55, edited by M. Abramowitz and I. A. Stegun (U.S. GPO, Washington, DC, 1965).

25 S. Uji, H. Shinagawa, T. Terashima, T. Yakabe, Y. Terai, M. Tokumoto, A. Kobayashi, H. Tanaka \& H. Kobayashi, Nature 410, 908 (2001)

${ }^{26}$ L. Balicas, J. S. Brooks, K. Storr, S. Uji, M. Tokumoto, H. Tanaka, H. Kobayashi, A. Kobayashi, V. Barzykin, and L. P. Gorkov Phys. Rev. Lett. 87, 067002 (2001).

27 V. Jaccarino and M. Peter, Phys. Rev. Lett. 9, 290 (1962). 